\title{
Suppression During Binocular Rivalry Broadens Orientation Tuning
}

\author{
Sam Ling and Randolph Blake \\ Vanderbilt University
}

\begin{abstract}
During binocular-rivalry suppression, an ordinarily visible stimulus is erased from awareness, but how is the sensory representation of that stimulus affected? Although it is established that rivalry suppression attenuates signal strength, the influence of suppression on signal fidelity remains unknown. Here, we show that noise plays a hitherto undiscovered role in the degradation of the percept under suppression. In Experiment 1, we measured psychometric functions for a stimulus presented under dominance and suppression, and found that the slope of these functions was shallower under suppression - a result suggesting that the signal representation was rendered noisier. Experiment 2 then revealed the source of this noise: An examination of the influence of suppression on the orientation bandwidth of noise masking showed that tuning bandwidth is significantly broadened under suppression. Thus, the discriminability of a suppressed stimulus is weakened not only by a general decrease in signal strength, but also by broader orientation tuning that introduces more noise in the neural representation of the suppressed stimulus.
\end{abstract}

When incompatible stimuli are presented separately to an observer's two eyes, the observer experiences oscillations in perception-a compelling phenomenon known as binocular rivalry (Wheatstone, 1838). During binocular rivalry, the stimulus presented to one eye may enjoy periods of dominance lasting several seconds; at the same time, the stimulus viewed by the other eye vanishes from visual awareness. This remarkable phenomenon has piqued the curiosity of psychophysicists and neuroscientists for well over a century (Alais \& Blake, 2005), and more recently some researchers have come to believe that rivalry may serve as a tool for studying the neural bases of visual awareness (e.g., C.Y. Kim \& Blake, 2005; Rees, Kreiman, \& Koch, 2002; Tong, 2003).

What is the fate of a suppressed stimulus during rivalry? Much of the evidence points to a sensory-based model for rivalry, according to which the neural representation of a suppressed stimulus is affected relatively early in visual processing. Psychophysically, it is well established that contrast sensitivity is impaired for probes presented to an eye during suppression (e.g., Blake \& Camisa, 1979; Nguyen, Freeman, \& Alais, 2003; Wales \& Fox, 1970). Neurophysiological and neuroimaging studies suggest that this temporary reduction in sensitivity is due to an attenuation of the neural response to the suppressed stimulus, an effect that has been observed at multiple stages within the visual hierarchy (e.g., Leopold \&

Copyright (@ 2009 Association for Psychological Science

Address correspondence to Sam Ling, Department of Psychology, Vanderbilt University, 611 Wilson Hall, Nashville, TN 37203 , s.ling@vanderbilt.edu.

\section{SUPPORTING INFORMATION}

Additional Supporting Information may be found in the on--line version of this article:

Figure S1

Figure S2

Please note: Wiley-Blackwell is not responsible for the content or functionality of any supporting materials supplied by the authors. Any queries (other than missing material) should be directed to the corresponding author for the article. 
Logothetis, 1996; Logothetis \& Schall, 1989; Polonsky, Blake, Braun, \& Heeger, 2000; Tong, Nakayama, Vaughan, \& Kanwisher, 1998; Wunderlich, Schneider, \& Kastner, 2005). Given these findings, it might seem that a stimulus fades from awareness simply because the neural strength of its signal has been "turned down." However, this may be only part of the story; one's ability to detect and discriminate a stimulus depends not only on the strength of the signal, but also on how noisy that signal is (Green \& Swets, 1966). Thus, although a decrease in the gain of a signal undoubtedly plays a role in rivalry suppression, degradation of the precision of that signal could also contribute to the impaired visibility of a suppressed stimulus-a possibility not yet explored. Physiologically, this degradation could correspond to a broadened tuning of the neural response that allows more irrelevant input to interfere with the signal response, resulting in more imprecise coding of the input stimulus.

In the study reported in this article, we explored whether suppression under binocular rivalry is brought about, in part, by an increase in signal noisiness. In the first experiment, we measured the influence of rivalry suppression on the slope of a psychometric function. We reasoned that if the stimulus representation becomes noisier under suppression, this should reveal itself through shallower slopes of the psychometric function. Indeed, we found that slopes became shallower under suppression, a result supporting the notion that signal noisiness plays an important role in perceptual degradation under rivalry. In the second experiment, we investigated the source of this noisiness. We used a noise-masking paradigm (Blake \& Holopigian, 1985; Legge \& Foley, 1980; Majaj, Pelli, Kurshan, \& Palomares, 2002; Solomon \& Pelli, 1994) to measure orientation tuning curves under dominance and suppression. Our aim was to learn whether suppression increases the noisiness of the neural representation of the suppressed stimulus by broadening its tuning curve. We discovered that suppression causes orientation tuning curves to broaden, which in turn renders a percept noisier.

\section{EXPERIMENT 1: NOISINESS OF ORIENTATION JUDGMENTS}

Does interocular suppression give rise to a noisier signal representation? One way a noisy representation would manifest itself is through shallow psychometric functions, as the slope of a psychometric function reveals the precision with which an observer makes a perceptual judgment. In our first experiment, we explored this potential perceptual consequence of rivalry suppression by measuring psychometric functions for an orientation discrimination task under states of interocular suppression and dominance.

\section{Method}

Four observers, including the first author, participated in Experiment 1. All had normal or corrected-to-normal vision.

Stimuli were generated on a G4 Power Macintosh running MATLAB and the Psychophysics Toolbox (Brainard, 1997; Pelli, 1997). Observers viewed the display in a darkened room on a gamma-corrected CRT (21-in. Sony MultiScan; refresh rate $=100 \mathrm{~Hz}$ ). Their heads were stabilized with a chin and forehead rest, $96 \mathrm{~cm}$ from the display. The display was viewed through a mirror stereoscope that presented the left half of the display exclusively to the left eye and the right half of the display exclusively to the right eye.

Throughout the experiment, each eye viewed a fixation point $\left(0.1^{\circ} \times 0.1^{\circ}\right)$, along with square fusion frames $\left(2^{\circ} \times 2^{\circ}\right)$ that helped stabilize binocular eye alignment (Fig. 1). In each trial, stimuli were presented dichoptically, with one eye viewing a bull's-eye pattern (3-cpd circular, sinusoidal grating of $10 \%$ contrast) and the other eye viewing a filtered noise patch (7.5\% root-mean-square contrast). The noise patch was low-pass spatial-frequency filtered (10-cpd cutoff), as well as bandpass filtered in the orientation domain $\left(20^{\circ}\right.$ bandwidth), with 
the center frequency of the noise band fixed at $0^{\circ}$ from vertical. The filter was smoothed in the Fourier domain to reduce Gibbs ringing artifacts that can arise from the presence of sharp boundaries in the Fourier representation of the stimulus.

To manipulate the dominance and suppression of these stimuli, we used the flash suppression technique (Wolfe, 1984): On each trial, the to-be-suppressed stimulus was presented monocularly for $2,500 \mathrm{~ms}$, after which time the competing stimulus (flash suppressor) abruptly appeared in the other eye, thereby suppressing perception of the initially presented stimulus in favor of the newly presented image. The timing and relatively small size of the competing stimuli were specifically chosen to maximize the duration of flash suppression, and to minimize instances of piecemeal rivalry within the probe duration (Hollins \& Hudnell, 1980). Figure 1 depicts a trial in which the bull's-eye stimulus appeared first in one eye, thus rendering the subsequent noise patch in the other eye dominant, and the bull's-eye suppressed (dominance condition). In the other half of the trials, the noise patch appeared first in one eye, rendering the bull's-eye stimulus in the other eye dominant, and the noise patch suppressed (suppression condition). One hundred milliseconds after the onset of the flash suppressor, a probe appeared briefly in the noise patch. So that the evoked dominance state would not be disrupted, the probe emerged and faded gradually over time, riding a Gaussian contrast ramp $(1,000 \mathrm{~ms})$. The probe was a tilted Gabor patch $\left(2^{\circ} \times 2^{\circ}\right.$, $40 \%$ contrast, $6 \mathrm{cpd}$ ), for which observers performed a two-alternative, forced-choice (2AFC) orientation discrimination task, reporting whether the patch was tilted to the right or left of vertical. To obtain orientation psychometric functions, we used the method of constant stimuli, varying the orientation of the Gabor $\left( \pm 0.2^{\circ}-15^{\circ}\right)$.

Each observer participated in a practice block of 50 trials, followed by nine experimental blocks of 100 trials each.

\section{Results}

Figure 2a shows 1 observer's psychometric functions for orientation discrimination measured under dominance and under suppression. The graph reveals a clear difference between the two conditions. To quantify this difference, we fit the psychometric functions with a cumulative Gaussian function from which we derived estimates of the slope $(\sigma)$ of those functions; greater values of $\sigma$ indicate shallower slopes. Slopes measured under suppression were markedly shallower than those measured under dominance (Fig. 2b). A $t$ test on the bootstrapped slope estimates (10,000 repetitions; Efron \& Tibshirani, 1993) confirmed that the slope differences between suppression and dominance were significant $(p s<.01)$. What is the basis for these differences?

One could argue that this slope decrease came about simply because observers sometimes could not see the probe under rivalry suppression at all, so that they guessed the orientation, which could flatten the psychometric function. Although it is unlikely that a $40 \%$ contrast probe would go entirely undetected in low-contrast noise, we conducted a control experiment to empirically rule out the possibility. Observers performed a two-interval, forced-choice detection task for stimuli under interocular suppression and dominance. The stimuli were similar to those used in Experiment 1, except that the probe Gabor, which was fixed at $0^{\circ}$ orientation, randomly appeared in only one of two successive intervals. If the change in slope in the main experiment occurred because observers could not see the stimulus under suppression, we would expect performance for the suppressed probe to be poor in this task. However, that was not the case; observers were very good at detecting the probe under suppression. Detection performance was above $90 \%$ accuracy for all 3 observers (S1: $94 \%$ in the dominance condition, $92 \%$ in the suppression condition; S2: $100 \%$ in the dominance condition, $92 \%$ in the suppression condition; S3: $98 \%$ in the dominance condition, $94 \%$ in the suppression condition). 
Alternatively, one could argue that probes, although sufficiently strong to be detected during suppression, were none-theless reduced in effective duration to a fraction of the actual 1-s exposure duration, so that discrimination was impaired. We tested this possibility with the same observers by measuring discrimination performance for probes presented for only 250 $\mathrm{ms}$ and found no change in the slopes of the orientation discrimination curves (results can be viewed in Fig. S1 in the Supporting Information available on-line-see p. 1355); this rules out reduced effective exposure duration as the cause of the poorer performance under suppression. So why did the slopes of the psychometric functions become shallower under suppression?

The slope of a psychometric function presumably reflects the precision of the sensory representation of a stimulus (Jazayeri \& Movshon, 2007). From this perspective, reductions in discriminability could arise from either a decrease in the signal strength or an increase in the signal's variability. To test whether our slope differences were attributable to an overall decrease in stimulus strength under rivalry suppression, we halved the contrast of the probe $(20 \%)$ and the noise (3.75\%), mimicking a reduction in stimulus strength under suppression, and collected orientation psychometric functions under monocular viewing. With the contrast of the stimulus halved, we observed no appreciable change in slope from the dominance condition (see Fig. 2b; $p$ s $>.05$ ). This finding implies that the slope changes during suppression were not the consequence of a reduction in effective contrast, but rather the result of a noisier stimulus representation. In the next experiment, we investigated the possible origin of increased noise during suppression.

\section{EXPERIMENT 2: ORIENTATION TUNING CURVES UNDER SUPPRESSION}

What is the source of the increased noise in the stimulus representation under suppression? An obvious possibility is that the tuning of orientation-selective filters is broadened, thereby increasing the range of orientations to which a given filter responds. Expanding the range of signals passed by a filter, in turn, should adversely affect the ability of that filter to signal small differences in orientation (Seung \& Sompolinsky, 1993). This degradation in precision would reveal itself psychophysically as a shallower psychometric function for an orientation discrimination task, which is what we found in Experiment 1. To pinpoint the noise source, we measured the impact of suppression on orientation tuning curves derived using a noisemasking technique (e.g., Blake \& Holopigian, 1985; Legge \& Foley, 1980; Majaj et al., 2002; Solomon \& Pelli, 1994). With noise masking, the more similar the features of a noise mask are to an embedded target stimulus, the greater the loss in sensitivity for detecting the target. This change in sensitivity as a function of noise content yields a psychophysical tuning curve, which allows one to infer the shape and sensitivity of the visual filter used to detect the target stimulus (Baldassi \& Verghese, 2005). In Experiment 2, our target stimulus was a vertically oriented Gabor patch, which was embedded in noise that varied in orientation content, ranging from the same orientation as the target to the orthogonal orientation. To assess whether the tuning bandwidth changed with rivalry state, we measured orientation tuning curves when the noise patch was dominant and when it was suppressed.

\section{Method}

Three observers, including the first author, participated in Experiment 2. Two of these observers (S1 and S2) had participated in the first experiment. All had normal or correctedto-normal vision.

As in Experiment 1, we manipulated the dominance and suppression of a noise patch with the flash suppression technique, using the same timing parameters and a similar stimulus configuration. The probe was a vertically oriented Gabor patch $\left(2^{\circ} \times 1.5^{\circ}, 6 \mathrm{cpd}\right)$ that 
appeared above or below fixation in a bed of filtered noise (15\% contrast). Observers performed a 2AFC location discrimination task, reporting whether the probe Gabor appeared above or below fixation.

We obtained perceptual tuning functions by using an orientation masking procedure, in which the orientations of the noise mask ranged from being identical to the orientation of the Gabor probe to being orthogonal to the orientation of the Gabor probe (Fig. 3a). The noise was bandpass-filtered in the orientation domain ( $20^{\circ}$ bandwidth), and the center frequency of this noise band could be $0^{\circ}, 8^{\circ}, 16^{\circ}, 24^{\circ}, 32^{\circ}, 40^{\circ}$, or $90^{\circ}$ from vertical. To prevent offchannel looking (e.g., Blake \& Holopigian, 1985), we symmetrically angled these noise bandpass orientations clockwise and counterclockwise relative to vertical. As in Experiment 1 , the noise patch was low-pass spatial-frequency filtered (10-cpd cutoff).

We used an adaptive staircase procedure (PEST, parameter estimation by sequential testing; Taylor \& Creelman, 1967) to estimate contrast thresholds at $75 \%$ performance for each noise bandpass orientation. The staircases for different noise-band-pass conditions were blocked, and their order pseudorandomized; staircases for the two states of rivalry were interleaved within each block. For each of the rivalry conditions, four thresholds were obtained per noise bandpass orientation.

\section{Results}

As is typically observed with noise masking, contrast thresholds were highest for noise that was most similar in orientation content to the probe $\left(0^{\circ}\right)$, tapering off as the difference in orientation between the probe and noise increased. Separate functions for suppression and dominance provided profiles of the tuning and sensitivity of the channel underlying the detection of the probe in these two conditions.

Under suppression, contrast thresholds were elevated across all noise orientations, a pattern supporting previous findings that the strength of a stimulus is attenuated under suppression (Blake \& Camisa, 1979; Nguyen et al., 2003; Wales \& Fox, 1970). However, the magnitude of this decrease in sensitivity was not uniform across bandpass orientations, which indicated a change in the shape of the perceptual tuning curve under suppression (Fig. 3b). To quantify the effects of suppression on the bandwidth of the tuning curves, we fit Gaussian functions to the data for each observer, assuming that the tuning curves were mirrorsymmetric and centered on $0^{\circ}$. Parameter estimates of the fitted Gaussian bandwidth revealed that suppression consistently increased the width of the tuning profile: Estimates of the bandwidth parameter (full-width at half-maximum) increased by around $15^{\circ}$ for all 3 observers (Fig. 3c). A $t$ test on the bootstrapped bandwidth estimates (10,000 repetitions; Efron \& Tibshirani, 1993) confirmed that the bandwidth differences between suppression and dominance were significant $(p s<.05)$.

Could the change in tuning we observed simply be attributed to rivalry suppression decreasing the strength of the noise in which the Gabor probe was embedded? To rule out the possibility that a reduction in the apparent noise contrast affected the shape of the tuning function, we conducted a control experiment in which the contrast of the noise was halved, emulating the decrease in effective contrast accompanying phases of interocular suppression (Blake, Tadin, Sobel, Chong, \& Raissian, 2006; Chong, Tadin, \& Blake, 2005). Observers performed the same task, only they viewed the stimuli monocularly, without a suppressor stimulus presented to the other eye. The noise-masking paradigm was used to measure orientation tuning curves for conditions in which the noise contrast was that of the original experiment (15\%) and in which the noise contrast was halved (7.5\%). If the contrast of the noise influenced tuning, one would expect tuning to be broadened in the latter condition. However, the tuning bandwidth remained the same regardless of the contrast of the noise ( $p$ s 
$>.05$; see Fig. S2 in the Supporting Information on-line). Thus, we attribute the changes in tuning in the main experiment to interocular suppression and not a reduction in effective contrast. These results, in other words, show that rivalry suppression not only weakens but also degrades the fidelity of the neural representation of the suppressed stimulus.

\section{DISCUSSION}

What could cause an ordinarily visible stimulus to vanish from visual awareness? Much of the recent psychophysical and neurophysiological evidence suggests that the strength of a stimulus is "turned down" under rivalry suppression (e.g., Blake \& Camisa, 1979; Blake et al., 2006; Freeman, Nguyen, \& Alais, 2005; Logothetis \& Schall, 1989; Polonsky et al., 2000; Tong, Meng, \& Blake, 2006; Wales \& Fox, 1970; Wunderlich et al., 2005). In the experiments reported here, we discovered another, hitherto undocumented, process that contributes to the perceptual degradation observed under rivalry. We found that psychometric functions became shallower under suppression, presumably because of the corrupted precision of observers' representation of the stimulus. Moreover, we were able to pinpoint the source of this perceptual degradation. Measuring orientation tuning curves under states of interocular dominance and suppression, we found broader orientation tuning under suppression, which suggests that the gain explanation of rivalry suppression tells only half of the story: The waning of a suppressed percept is also due to a decrease in the fidelity of the stimulus representation. In recent years, noise has come to play a rather prominent role in models aimed at explaining the stochastic alternations in dominance during binocular rivalry (Brascamp, van Ee, Noest, Jacobs, \& van den Berg, 2006; Y.-J. Kim, Grabowecky, \& Suzuki, 2006; Moreno-Bote, Rinzel, \& Rubin, 2007). Our work presented here implies that noise exerts an even more pervasive effect on rivalry, by degrading the neural representation of a suppressed stimulus and, thereby, contributing to its temporary disadvantage in the competition for visual awareness.

Recently, Baker and Meese (2007) measured orientation tuning psychophysically under dichoptic masking. They measured tuning curves when a target in one eye was in phase with a similar mask stimulus in the other eye, causing binocular summation, and when the target and mask were in antiphase, causing binocular suppression. They reported sharper orientation tuning in the summation phase than in the suppression phase. These findings may be related to ours, as some aspects of dichoptic masking are shared with rivalry (van Boxtel, van Ee, \& Erkelens, 2007). The interocular suppression technique Baker and Meese used to probe dichoptic masking is similar to the flash suppression technique, except that with dichoptic masking, the dominance of one eye or the other is not tipped toward a particular stimulus by initial monocular presentation. It is possible that the mechanisms causing the broadened tuning Baker and Meese observed for dichoptic antiphase stimuli are similar to those underlying the broadened tuning we observed for rivalry suppression.

How does this broadened tuning arise? One potential source could be a broadened tuning of individual detectors in cortical areas such as V1. One of the prevailing accounts of orientation selectivity at the neural level is the modified feed-forward model (e.g., Ferster \& Miller, 2000), which describes broad orientation tuning as a product of feed-forward excitatory afferents from cells in the lateral geniculate nucleus (LGN) to cells in V1 that are arranged spatially in an orientation-specific manner. How would we explain our results under this model? Broadened tuning modulation of the excitatory component could arise from a change in the spatial arrangement of the afferent connections from LGN to simple cells in V1, such that the subfield aspect ratio of these excitatory inputs decreases: The smaller the subfield aspect ratio, the broader the tuning. Thus, it is possible that feedback from cortical areas to the LGN causes a change in the spatial arrangement of the thalamocortical inputs, broadening tuning at the individual neural level. 
Alternatively, the broadened orientation tuning we observed could be the result of changes at the level of the neural population response. It is possible that our results are not due to changes in bandwidth for individual detectors tuned to orientation, but rather are due to a selective change in the neural sensitivity of those detectors. Population responses are shaped by lateral interactions within cortex; responses of orientation-selective neurons are influenced by activity in other neurons not tuned to the same stimulus (Blakemore \& Tobin, 1972). Perhaps these intracortical lateral interactions are weakened by suppression: When a stimulus of a given orientation is suppressed, such weakening could cause detectors responding to flanking orientations to respond more strongly. Ordinarily, activity in orientation-tuned neurons would exert inhibition on other orientation detectors, but when a particular orientation is suppressed under rivalry, there might be a release from inhibition that produces more activity in neurons not tuned to that orientation. This would lead to a broadening of the population response to a given stimulus orientation, effectively introducing noise into the sensory representation of that orientation and thereby weakening the percept of an oriented stimulus.

Where in the visual pathway do these effects occur? Although numerous studies have explored the effect of rivalry suppression on the amplitude of neural responses across visual areas (e.g., Logothetis \& Schall, 1989; Polonsky et al., 2000; Tong et al., 1998; Wunderlich et al., 2005), to our knowledge no one has physiologically measured how tuning is affected by rivalry. One currently popular theory posits that the neural events underlying rivalry are distributed throughout much of the visual hierarchy (e.g., Tong et al., 2006), and studies have demonstrated effects ranging from subcortical modulation of rivalry in the LGN (Haynes, Deichmann, \& Rees, 2005; Wunderlich et al., 2005) to modulation in higher ventral-stream areas (e.g., Tong et al., 1998) believed to be involved in higher-level object processing. It would be difficult to explain the broadening of orientation tuning during suppression on the basis of precortical mechanisms because neurons in the LGN are not tuned to orientation (Hubel \& Wiesel, 1961), and are therefore incapable of carrying out the orientation-specific computations that yielded our results. Of course, we cannot rule out the possibility that interocular inhibition within the LGN contributes, at least partially, to the overall reduction in contrast sensitivity during suppression. Although the effect of rivalry on orientation tuning appears to implicate early cortical visual areas (e.g., V1) as a source of rivalry modulation, it is still possible that modulation continues to grow in strength throughout the visual stream (Nguyen et al., 2003). Indeed, it may be that the selectivity of more complex neural representations in other visual areas also undergoes broadening of tuning during suppression.

\section{Supplementary Material}

Refer to Web version on PubMed Central for supplementary material.

\section{Acknowledgments}

We thank Denis Pelli, Joel Pearson, and two anonymous reviewers for their helpful comments on an earlier version of this article. This research was funded by National Institutes of Health (NIH) Grant EY13358. S.L. is supported by NIH Training Grant EY007135.

\section{REFERENCES}

Alais, D.; Blake, R., editors. Binocular rivalry. London: MIT Press; 2005.

Baker DH, Meese TS. Binocular contrast interactions: Dichoptic masking is not a single process. Vision Research. 2007; 47:3096-3107. [PubMed: 17904610] 
Baldassi S, Verghese P. Attention to locations and features: Different top-down modulation of detector weights. Journal of Vision. 2005; 5(6) Article 7. Retrieved September 12, 2008, from http:// www.journalofvision.org/5/6/7/.

Blake R, Camisa J. On the inhibitory nature of binocular rivalry suppression. Journal of Experimental Psychology: Human Perception and Performance. 1979; 5:315-323. [PubMed: 528942]

Blake R, Holopigian K. Orientation selectivity in cats and in humans assessed by masking. Vision Research. 1985; 25:1459-1468. [PubMed: 4090280]

Blake, R.; Tadin, D.; Sobel, K.; Chong, SC.; Raissian, R. Strength of early visual adaptation depends on visual awareness; Proceedings of the National Academy of Sciences; USA. 2006. p. 4783-4788.

Blakemore C, Tobin EA. Lateral inhibition between orientation detectors in the cat's visual cortex. Experimental Brain Research. 1972; 15:439-440.

Brainard DH. The Psychophysics Toolbox. Spatial Vision. 1997; 10:433-436. [PubMed: 9176952]

Brascamp JW, van Ee R, Noest AJ, Jacobs R, van den Berg AV. The time course of binocular rivalry reveals a fundamental role of noise. Journal of Vision. 2006 6(11) Article 8, from http:// www.journalofvision.org/6/11/8/.

Chong SC, Tadin D, Blake R. Endogenous attention prolongs dominance durations in binocular rivalry. Journal of Vision. 2005 5(11) Article 6, from http://www.journalofvision.org/5/11/6/.

Efron, B.; Tibshirani, RJ. An introduction to the bootstrap. New York: Chapman and Hall; 1993.

Ferster D, Miller KD. Neural mechanisms of orientation selectivity in the visual cortex. Annual Review of Neuroscience. 2000; 23:441-471.

Freeman, AW.; Nguyen, VA.; Alais, D. The nature and depth of binocular rivalry suppression. In: Alais, D.; Blake, R., editors. Binocular rivalry. London: MIT Press; 2005. p. 47-62.

Green, DM.; Swets, JA. Signal detection theory and psychophysics. New York: Wiley; 1966.

Haynes JD, Deichmann R, Rees G. Eye-specific effects of binocular rivalry in the human lateral geniculate nucleus. Nature. 2005; 438:496-499. [PubMed: 16244649]

Hollins M, Hudnell K. Adaptation of the binocular rivalry mechanism. Investigative Ophthalmology \& Visual Science. 1980; 19:1117-1120. [PubMed: 7410003]

Hubel DH, Wiesel TN. Integrative action in the cat's lateral geniculate body. Journal of Physiology. 1961; 155:385-398. [PubMed: 13716436]

Jazayeri M, Movshon JA. A new perceptual illusion reveals mechanisms of sensory decoding. Nature. 2007; 446:912-915. [PubMed: 17410125]

Kim CY, Blake R. Psychophysical magic: Rendering the visible "invisible.”. Trends in Cognitive Sciences. 2005; 9:381-388. [PubMed: 16006172]

Kim Y-J, Grabowecky M, Suzuki S. Stochastic resonance in binocular rivalry. Vision Research. 2006; 46:392-406. [PubMed: 16183099]

Legge GE, Foley JM. Contrast masking in human vision. Journal of the Optical Society of America. 1980; 70:1458-1471. [PubMed: 7463185]

Leopold D, Logothetis N. Activity changes in early visual cortex reflect monkeys' percepts during binocular rivalry. Nature. 1996; 379:549-553. [PubMed: 8596635]

Logothetis NK, Schall JD. Neuronal correlates of subjective visual perception. Science. 1989; 245:761-763. [PubMed: 2772635]

Majaj NJ, Pelli DG, Kurshan P, Palomares M. The role of spatial frequency channels in letter identification. Vision Research. 2002; 42:1165-1184. [PubMed: 11997055]

Moreno-Bote R, Rinzel J, Rubin N. Noise-induced alternations in an attractor network model of perceptual bistability. Journal of Neurophysiology. 2007; 98:1125-1139. [PubMed: 17615138]

Nguyen VA, Freeman AW, Alais D. Increasing depth of binocular rivalry suppression along two visual pathways. Vision Research. 2003; 43:2003-2008. [PubMed: 12842153]

Pelli DG. The VideoToolbox software for visual psychophysics: Transforming numbers into movies. Spatial Vision. 1997; 10:437-442. [PubMed: 9176953]

Polonsky A, Blake R, Braun J, Heeger DJ. Neuronal activity in human primary visual cortex correlates with perception during binocular rivalry. Nature Neuroscience. 2000; 3:1153-1159.

Rees G, Kreiman G, Koch C. Neural correlates of consciousness in humans. Nature Reviews Neuroscience. 2002; 3:261-270. 
Seung, HS.; Sompolinsky, H. Simple models for reading neuronal population codes; Proceedings of the National Academy of Sciences; USA. 1993. p. 10749-10753.

Solomon JA, Pelli DG. The visual filter mediating letter identification. Nature. 1994; 369:395-397. [PubMed: 8196766]

Taylor MM, Creelman CD. PEST: Efficient estimates of probability functions. Journal of the Acoustical Society of America. 1967; 41:782-787.

Tong F. Primary visual cortex and visual awareness. Nature Reviews Neuroscience. 2003; 4:219-229.

Tong F, Meng M, Blake R. Neural bases of binocular rivalry. Trends in Cognitive Sciences. 2006; 10:502-511. [PubMed: 16997612]

Tong F, Nakayama K, Vaughan JT, Kanwisher N. Binocular rivalry and visual awareness in human extrastriate cortex. Neuron. 1998; 21:753-759. [PubMed: 9808462]

van Boxtel JJA, van Ee R, Erkelens CJ. Dichoptic masking and binocular rivalry share common perceptual dynamics. Journal of Vision. 2007 7(14) Article 3, from http://journalofvision.org/ $7 / 14 / 3 /$.

Wales NJ, Fox R. Increment detection thresholds during binocular rivalry suppression. Perception \& Psychophysics. 1970; 8:90-94.

Wheatstone C. Contributions to the physiology of vision: Part the first. On some remarkable, and hitherto unobserved, phenomena of binocular vision. Philosophical Transactions of the Royal Society of London. 1838; 128:371-394.

Wolfe JM. Reversing ocular dominance and suppression in a single flash. Vision Research. 1984; 24:471-478. [PubMed: 6740966]

Wunderlich K, Schneider KA, Kastner S. Neural correlates of binocular rivalry in the human lateral geniculate nucleus. Nature Neuroscience. 2005; 8:1595-1602. 


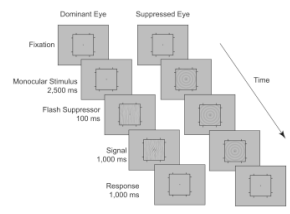

Fig. 1.

Example of a display used in Experiment 1. Two competing stimuli were viewed dichoptically: a bull's-eye and a stimulus consisting of bandpass-filtered noise. To control rivalry state, we used the flash suppression technique. Following flash-induced dominance/ suppression, a small, tilted Gabor patch appeared briefly within the noise patch, and observers reported the direction of tilt of the Gabor patch (two-alternative, forced-choice task). 


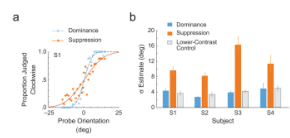

Fig. 2.

The influence of rivalry suppression on orientation psychometric functions (Experiment 1). The example psychometric function (a) shows the proportion of trials on which observer S1 judged the probe to be tilted clockwise as a function of the orientation of the probe, separately for the dominance and suppression conditions. The bar graph (b) presents estimates of the slopes of the psychometric functions for all 4 observers. Results are shown for the dominance and suppression conditions, as well as for a control experiment in which the contrast of the noise and probe was halved. Shallower slopes (greater values of $\sigma$ ) imply a noisier representation of the probe orientation. Error bars correspond to bootstrapped $95 \%$ confidence intervals. 


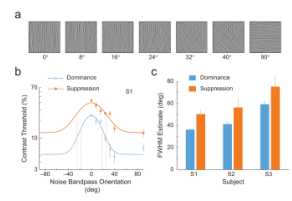

Fig. 3.

The influence of rivalry suppression on orientation tuning (Experiment 2). To obtain perceptual tuning functions, we used an orientation-bandpass noise-masking procedure (a) in which the noise patch and Gabor probe ranged from being identical in orientation $\left(0^{\circ}\right)$ to being orthogonal in orientation $\left(90^{\circ}\right)$. The probe grating was vertical, and the orientation content of the noise patch varied (in the illustrations, similarity between the probe and noise patch decreases from left to right). In the example orientation tuning curve (b), the contrast thresholds are shown separately for the dominance and suppression conditions. The data, from subject S1, were fit with a modified Gaussian function. The drop-down dotted lines correspond to the estimates of the bandwidth parameter (full-width at half-maximum, or FWHM). Error bars correspond to $\pm 1 S E$. The bar graph (c) presents bandwidth parameter estimates (FWHM) in the dominance and suppression conditions for each observer. Error bars correspond to bootstrapped $95 \%$ confidence intervals. 